\title{
ON THE WEYL AND RICCI TENSORS OF GENERALIZED ROBERTSON-WALKER SPACE-TIMES
}

\author{
CARLO ALBERTO MANTICA AND LUCA GUIDO MOLINARI
}

\begin{abstract}
We prove theorems about the Ricci and the Weyl tensors on generalized Robertson-Walker space-times of dimension $n \geq 3$. In particular, we show that the concircular vector introduced by Chen decomposes the Ricci tensor as a perfect fluid term plus a term linear in the contracted Weyl tensor. The Weyl tensor is harmonic if and only if it is annihilated by Chen's vector, and any of the two conditions is necessary and sufficient for the GRW space-time to be quasi-Einstein (perfect fluid) manifold. Finally, the general structure of the Riemann tensor for Robertson-Walker space-times is given, in terms of Chen's vector. A GRW space-time in $n=4$ with null conformal divergence is a Robertson-Walker space-time.
\end{abstract}

\section{INTRODUCTION}

The beautiful theorem by Chen (2014, 1] gives a simple and covariant characterisation of Generalized Robertson-Walker space-times (GRW). It states that a Lorentzian manifold of dimension $n \geq 3$ is a GRW if and only if there exists a vector field $X_{j}$, which we name Chen's vector, such that $X^{j} X_{j}<0$ and

$$
\nabla_{j} X_{k}=\rho g_{j k}
$$

where $\rho$ is some scalar field. The equation is the defining property of a concircular vector, introduced by Fialkow [2] (Yano gives the same name to a broader class of vectors [3]). The existence of such a vector field is a necessary and sufficient condition for a local expression of the metric with the warped form

$$
d s^{2}=-d t^{2}+q^{2}(t) g_{\mu \nu}^{*}(\vec{x}) d x^{\mu} d x^{\nu}
$$

where $g_{\mu \nu}^{*}$ is the metric tensor of a Riemannian submanifold $M^{*}$ parametrised by $\vec{x}$. The local form (2) is actually how GRW manifolds were defined in 1995 by Alias et al. [4, and studied subsequently (see for example [5, 6, 7).

The strong property of Chen's vector upon differentiation allows for a determination of several interesting properties of GRW manifolds. The following two sections are devoted to the Ricci and to the Weyl tensor respectively. After showing that Chen's vector is an eigenvector of the Ricci tensor, the Ricci tensor is expressed as the sum of a perfect fluid term and a term proportional to the Weyl tensor.

The main theorem states that the Weyl tensor is harmonic $\left(\nabla^{m} C_{j k l m}=0\right)$ if and only if $C_{j k l m} X^{m}=0$. Any of the two conditions is necessary and sufficient for the GRW manifold to be a quasi-Einstein (or perfect fluid) space-time, i.e. for the Ricci tensor to have the form $R_{i j}=A g_{i j}+B u_{i} u_{j}$.

Date: 31 july 2016.

2010 Mathematics Subject Classification. Primary 53B30, 53B50, Secondary 53C80, 83C15.

Key words and phrases. Lorentzian manifold, Generalized Robertson-Walker manifold, harmonic Weyl tensor, concircular vector. 
Part of these statements existed in the literature: in 1994 Gebarowski [8] proved that the fibers of a GRW space-time are Einstein (i.e. the Ricci tensor of the sub-manifold $M^{*}$ in the decomposition (2) has the form $R_{\mu \nu}^{*}=R^{*} g_{\mu \nu} /(n-1)$ ) if and only if $\nabla^{m} C_{j k l m}=0$. Then, Sanchez (1999, 9]) stated that the fibers of a GRW space-time are Einstein if and only if the GRW manifold is quasi-Einstein (or perfect-fluid). Recently, Mantica et al. (2016, [10]) showed the converse: a perfectfluid Lorentzian manifold with $\nabla^{m} C_{j k l m}=0$ is a GRW space-time (see also [11]). In this presentation, besides other results, we show that the same propositions hold with the algebraic condition $C_{j k l m} X^{m}=0$ where $X$ is Chen's vector.

In the last section we consider conformally flat GRW space-times, i.e. RobertsonWalker space-times, and provide the general form of the Riemann tensor. In particular, the aforementioned results imply that in $n=4$ a conformally harmonic GRW space-time is a Robertson-Walker space-time.

\section{The RiCCI TENSOR}

Theorem 2.1. On a GRW manifold:

1) Chen's vector $X_{j}$ is an eigenvector of the Ricci tensor,

$$
R_{j m} X^{m}=\xi X_{j},
$$

and the following holds for the eigenvalue:

$$
\xi=-(n-1) \frac{X^{k} \nabla_{k} \rho}{X^{2}}, \quad \nabla_{i} \xi=\theta X_{i}
$$

where $\theta$ is a scalar field.

2) the Ricci tensor can be expressed in terms of the Weyl tensor, the curvature scalar $R$, the eigenvalue $\xi$ and Chen's vector:

$$
R_{k l}=(n-2) C_{j k l m} \frac{X^{j} X^{m}}{X^{2}}+\frac{R-\xi}{n-1}\left(g_{k l}-\frac{X_{k} X_{l}}{X^{2}}\right)+\xi \frac{X_{k} X_{l}}{X^{2}} ;
$$

Proof. By Chen's theorem, on a GRW manifold there is a vector field $X_{j}$ such that $\nabla_{i} X_{j}=\rho g_{i j}$. Then $\left[\nabla_{i}, \nabla_{j}\right] X_{k}=\left(\nabla_{i} \rho\right) g_{j k}-\left(\nabla_{j} \rho\right) g_{i k}$ i.e. $R_{i j k}{ }^{m} X_{m}=$ $\left(\nabla_{i} \rho\right) g_{j k}-\left(\nabla_{j} \rho\right) g_{i k}$. A contraction with $g^{j k}$ gives $R_{i m} X^{m}=-(n-1) \nabla_{i} \rho$, while the contraction with $X^{k}$ gives $0=X_{j} \nabla_{i} \rho-X_{i} \nabla_{j} \rho$, then $X^{2} \nabla_{j} \rho=X_{j}\left(X^{i} \nabla_{i} \rho\right)$ i.e. $\nabla_{i} \rho$ is proportional to $X_{i}$. Therefore (3) follows.

Moreover:

$$
R_{i j k}{ }^{m} X_{m}=-\frac{\xi}{n-1}\left(X_{i} g_{j k}-X_{j} g_{i k}\right)
$$

The derivative of (6) is

$$
X^{m} \nabla_{s} R_{i j k m}+\rho R_{i j k s}=-\frac{\nabla_{s} \xi}{n-1}\left(X_{i} g_{j k}-X_{j} g_{i k}\right)-\frac{\rho \xi}{n-1}\left(g_{s i} g_{j k}-g_{i k} g_{j s}\right),
$$

the sum on cyclic permutations of indices sij and the second Bianchi identity give:

$$
0=g_{j k}\left(X_{i} \nabla_{s} \xi-X_{s} \nabla_{i} \xi\right)+g_{i k}\left(X_{s} \nabla_{j} \xi-X_{j} \nabla_{s} \xi\right)+g_{s k}\left(X_{j} \nabla_{i} \xi-X_{i} \nabla_{j} \xi\right) .
$$

The contraction with $g^{s k}$ finally gives $X_{j} \nabla_{i} \xi-X_{i} \nabla_{j} \xi=0$, with solution $\nabla_{i} \xi=\theta X_{i}$, thus proving (4).

By definition, the Weyl tensor is

$$
C_{j k l m}=R_{j k l m}+\frac{1}{n-2}\left(g_{j m} R_{k l}-g_{k m} R_{j l}+R_{j m} g_{k l}-R_{k m} g_{j l}\right)-R \frac{g_{j m} g_{k l}-g_{j l} g_{k m}}{(n-1)(n-2)}
$$


A contraction with $X^{m}$ and (6) give:

$$
C_{j k l}{ }^{m} X_{m}=\frac{\xi-R}{(n-1)(n-2)}\left(X_{j} g_{k l}-X_{k} g_{j l}\right)+\frac{1}{n-2}\left(X_{j} R_{k l}-X_{k} R_{j l}\right) \text {. }
$$

Another contraction with $X^{j}$ gives the Ricci tensor (5).

A covariant derivative of the eigenvalue equation (3), and use of (11) and $\nabla^{k} R_{k j}=$ $\frac{1}{2} \nabla_{j} R$, where $R$ is the curvature scalar, gives a relation that will be important in the sequel:

$$
\frac{1}{2} X^{k} \nabla_{k} R=n \rho \xi-\rho R+X^{2} \theta
$$

Multiplication of (6) by $X_{l}$ and summation on cyclic permutations of $i j l$ shows that Chen's vector is "Riemann compatible" [1]: $X_{i} X^{m} R_{j l k m}+X_{j} X^{m} R_{l i k m}+$ $X_{l} X^{m} R_{i j k m}=0$. In general, Riemann implies Weyl compatibility [13]. In the present case (it can be checked with (7)):

$$
X_{i} X^{m} C_{j k l m}+X_{j} X^{m} C_{k i l m}+X_{k} X^{m} C_{i j l m}=0
$$

\section{The WeYL Tensor}

We now focus on the Weyl tensor. It is useful to introduce the auxiliary symmetric trace-less tensor

$$
\mathrm{C}_{j k}=C_{a j k b} \frac{X^{a} X^{b}}{X^{2}}
$$

Note the properties $X^{j} C_{j k}=0$ and $X^{j} \nabla_{l} C_{j k}=-\left(\nabla_{l} X^{j}\right) C_{j k}=-\rho C_{k l}$, that will be frequently used.

The contraction of (9) by $X^{i}$ or, the insertion of the expression of the Ricci tensor in (7), gives

Proposition 3.1. The Weyl tensor of a GRW manifold satisfies the identity:

$$
C_{j k l m} X^{m}=X_{j} \mathrm{C}_{k l}-X_{k} \mathrm{C}_{j l}
$$

It implies that $C_{j k l m} X^{m}=0$ if and only if $\mathrm{C}_{k l}=0$.

The general expression for the covariant divergence of the Weyl tensor is:

$$
\nabla^{m} C_{j k l m}=-\frac{n-3}{n-2}\left[\nabla_{j} R_{k l}-\nabla_{k} R_{j l}-\frac{g_{k l} \nabla_{j} R-g_{j l} \nabla_{k} R}{2(n-1)}\right] .
$$

We look for an expression in terms of the contracted tensor $C_{j k}$. The following covariant derivatives are evaluated with (1) and $\nabla_{j} \xi=\theta X_{j}$ :

$$
\begin{aligned}
\nabla_{j} R_{k l}-\nabla_{k} R_{j l} & =(n-2)\left(\nabla_{j} \mathrm{C}_{k l}-\nabla_{k} \mathrm{C}_{j l}\right) \\
& +\frac{1}{n-1}\left(n \rho \xi-\rho R+X^{2} \theta\right)\left(\frac{X_{k}}{X^{2}} g_{j l}-\frac{X_{j}}{X^{2}} g_{k l}\right) \\
& +\frac{1}{n-1}\left[\left(g_{k l}-\frac{X_{k} X_{l}}{X^{2}}\right) \nabla_{j} R-\left(g_{j l}-\frac{X_{j} X_{l}}{X^{2}}\right) \nabla_{k} R\right] \\
& =\frac{1}{n-1}\left[\left(g_{k l}-\frac{X_{k} X_{l}}{X^{2}}\right) \nabla_{j} R-\left(g_{j l}-\frac{X_{j} X_{l}}{X^{2}}\right) \nabla_{k} R\right. \\
& \left.+\frac{1}{2}\left(\frac{X_{k} X_{s}}{X^{2}} g_{j l}-\frac{X_{j} X_{s}}{X^{2}} g_{k l}\right) \nabla^{s} R\right]+(n-2)\left(\nabla_{j} C_{k l}-\nabla_{k} \mathrm{C}_{j l}\right)
\end{aligned}
$$


because of the identity (8). Eq. (11) becomes:

$$
\begin{aligned}
& \nabla^{m} C_{j k l m}=-(n-3)\left(\nabla_{j} C_{k l}-\nabla_{k} C_{j l}\right)+\frac{n-3}{(n-1)(n-2)} \frac{X_{l}}{X^{2}}\left(X_{k} \nabla_{j} R-X_{j} \nabla_{k} R\right) \\
& -\frac{n-3}{2(n-1)(n-2)}\left[g_{k l}\left(g_{j m}-\frac{X_{j} X_{m}}{X^{2}}\right) \nabla^{m} R-g_{j l}\left(g_{k m}-\frac{X_{k} X_{m}}{X^{2}}\right) \nabla^{m} R\right]
\end{aligned}
$$

Its contraction with $g^{k l}$ gives

$$
\nabla^{k} C_{j k}=\frac{n-3}{2(n-1)(n-2)}\left(\nabla_{j} R-\frac{X_{j} X^{l}}{X^{2}} \nabla_{l} R\right) .
$$

\section{Lemma 3.2.}

$$
X^{j} \nabla_{j} C_{k l}=-2 \rho C_{k l}
$$

Proof. The contraction of (13) with $X^{j}$ is:

$$
X^{j} \nabla^{m} C_{j k l m}=-(n-3)\left(X^{j} \nabla_{j} C_{k l}+\rho C_{k l}\right)-\frac{(n-3) X_{l}}{2(n-1)(n-2)}\left(\nabla_{k} R-\frac{X_{k} X^{j}}{X^{2}} \nabla_{j} R\right)
$$

With the aid of (14) and with a permutation of indices, it becomes:

$$
\nabla^{j}\left(C_{j l k m} X^{m}\right)=-(n-3)\left(X^{j} \nabla_{j} C_{k l}+\rho C_{k l}\right)-X_{l} \nabla^{m} C_{k m}
$$

The left-hand-side of this equation is evaluated by means of (10): $\nabla^{j}\left(C_{j l k m} X^{m}\right)=$ $\nabla^{j}\left(X_{j} \mathrm{C}_{l k}-X_{l} \mathrm{C}_{j k}\right)$ i.e.

$$
\nabla^{j}\left(C_{j l k m} X^{m}\right)=(n-1) \rho C_{k l}+X^{j} \nabla_{j} C_{k l}-X_{l} \nabla^{j} C_{j k}
$$

The two equations imply (15).

The following statement is important:

Proposition 3.3. If $X^{l} \nabla^{m} C_{j k l m}=0$ then:

$$
\begin{gathered}
\nabla_{i} R=X_{i} \frac{X^{m} \nabla_{m}}{X^{2}} R \\
\nabla^{m} C_{j k l m}=-(n-3)\left(\nabla_{j} \mathrm{C}_{k l}-\nabla_{k} \mathrm{C}_{j l}\right)
\end{gathered}
$$

Proof. Recall that $X^{l} \nabla_{j} C_{k l}=-\rho C_{j k}$. The contraction of (13) with $X^{l}$ gives

$$
X^{l} \nabla^{m} C_{j k l m}=\frac{n-3}{2(n-1)(n-2)}\left(X_{k} \nabla_{j} R-X_{j} \nabla_{k} R\right)
$$

If $X^{l} \nabla^{m} C_{j k l m}=0$ then $X_{k} \nabla_{j} R=X_{j} \nabla_{k} R$, with solution (18). Eq.(13) greatly simplifies and reduces to (19).

Since $X^{l} \nabla^{m} C_{j k l m}=\nabla^{m}\left(C_{j k l m} X^{l}\right)$, the proposition holds in particular if $\nabla^{m} C_{j k l m}=$ 0 or if $X^{m} C_{j k l m}=0$.

We are ready to prove the main theorem:

Theorem 3.4. On a GRW space-time with Chen vector $X_{j}$

$$
C_{j k l m} X^{m}=0 \quad \Longleftrightarrow \quad \nabla^{m} C_{j k l m}=0
$$


Proof. If $C_{j k l m} X^{m}=0$ then $C_{j k}=0$. The right-hand-side of (19) is zero, and the Weyl tensor is harmonic.

If $\nabla^{m} C_{j k l m}=0$ then $\nabla_{j} C_{k l}-\nabla_{k} C_{j l}=0$. In particular:

$$
X^{j} \nabla_{j} \mathrm{C}_{k l}=-\rho \mathrm{C}_{k l}
$$

Because of (15) it is $C_{k l}=0$ i.e. $C_{j k l m} X^{m}=0$.

Proposition 3.5. On a GRW space-time with Chen vector $X_{k}, C_{j k l m} X^{m}=0$ if and only if

$$
R_{j k}=\alpha g_{j k}+\beta \frac{X_{j} X_{k}}{X^{2}}
$$

for suitable scalars $\alpha$ and $\beta$.

Proof. If $C_{j k l m} X^{m}=0$, then (5) gives $R_{j k}$ the perfect fluid form. If $R_{j k}=\alpha g_{j k}+$ $\beta \frac{X_{j} X_{k}}{X^{2}}$ then $R=n \alpha+\beta$ and $\xi=\alpha+\beta$, and the left hand side of (7) is zero.

Because of the main theorem 3.4 we also have:

Proposition 3.6. On a GRW space-time, it is $\nabla^{m} C_{j k l m}=0$ if and only if the Ricci tensor has the structure (23) (i.e. the manifold is quasi-Einstein).

\section{Robertson-WALKER SPACE-TIMES}

Robertson-Walker space-times are an important subclass of GRW space-times; they share the property of being conformally flat, $C_{j k l m}=0$. Let us then consider GRW space-times that are conformally flat.

The following theorem applies [16: A GRW space-time is conformally flat if and only if the GRW manifold is the ordinary Robertson-Walker space-time (or: if and only if the sub-manifold $M^{*}$ in the warped product (2) is a space of constant curvature).

With $C_{j k l m}=0$ the Ricci tensor has the structure (23) and, in view of theorem 3.4 and proposition 3.5 the Riemann tensor is largely determined:

$$
\begin{aligned}
& R_{j k l m}=\frac{2 \xi-R}{(n-1)(n-2)}\left(g_{k l} g_{j m}-g_{k m} g_{j l}\right) \\
& +\frac{R-n \xi}{(n-1)(n-2)}\left[g_{j m} \frac{X_{k} X_{l}}{X^{2}}-g_{k m} \frac{X_{j} X_{l}}{X^{2}}+g_{k l} \frac{X_{j} X_{m}}{X^{2}}-g_{j l} \frac{X_{k} X_{m}}{X^{2}}\right]
\end{aligned}
$$

where $X$ is Chen's vector, $R$ is the curvature scalar, $\xi$ is the eigenvalue of the Ricci tensor with eigenvector $X$.

Eq. (24) is the general form of the Riemann tensor of a Robertson-Walker spacetime. The form characterises manifolds of quasi-constant curvature, introduced by Chen and Yano in 1972, [15].

In four-dimensions, the Weyl tensor on a pseudo-Riemannian manifold has a special property: if $C_{j k l m} u^{m}=0$, where $u^{k} u_{k} \neq 0$, then $u_{i} C_{j k l m}+u_{j} C_{k i l m}+$ $u_{k} C_{i j l m}=0$ (see [14 page 128). In particular, a contraction with $u^{i}$ gives $C_{j k l m}=$ 0 . As a consequence we may state:

Proposition 4.1. In $n=4$, a GRW manifold with $\nabla^{m} C_{j k l m}=0$ is a RobertsonWalker space-time.

Proof. In a GRW the condition $\nabla^{m} C_{j k l m}=0$ is equivalent to $C_{j k l m} X^{m}=0$. Then, in $n=4$, it is $C_{j k l m}=0$. 


\section{REFERENCES}

[1] B-Y. Chen, A simple characterization of generalized Robertson-Walker manifolds, Gen. Relativ. Gravit. 46 (2014) 1833.

[2] A. Fialkow, Conformal geodesics, Transactions of the American Mathematical Society 45 n.3 (1939), 443-473.

[3] K. Yano, On the torse-forming directions in Riemannian spaces, Proc. Imp. Acad. Tokyo, 20 (1944) 340-345.

[4] L. J. Alías, A. Romero, M. Sánchez, Uniqueness of complete spacelike hypersurfaces of constant mean curvature in generalized Robertson-Walker space-times, Gen. Relativ. Gravit. 27 n.1 (1995) 71-84.

[5] M. Sánchez, On the geometry of generalized Robertson-Walker spacetimes: geodesics, Gen. Relativ. Gravit. 30 (1998) 915-932.

[6] R. Deszcz, and M. Kucharski, On curvature properties of certain generalized RobertsonWalker space-times, Tsukuba J. Math. 23 n.1 (1999), 113-130.

[7] M. Gutíerrez and B. Olea, Global decomposition of a Lorentzian manifold as a Generalized Robertson-Walker space, Differential Geom. Appl. 27 (2009) 146-156.

[8] A. Gebarowski, On nearly conformally symmetric warped product spacetimes, Soochow J. Math. 20 n.1 (1994) 61-75.

[9] M. Sánchez, On the geometry of generalized Robertson-Walker spacetimes: curvature and Killing fields, Gen. Relativ. Gravit. 31 (1999) 1-15.

[10] C. A. Mantica, L. G. Molinari and U. C. De, A condition for a perfect-fluid space-time to be a generalized Robertson-Walker space-time, J. Math. Phys. 57 (2016), 022508 (6pp.); Erratum, J. Math.Phys. 57 (2016) 049901.

[11] C. A. Mantica, Y. J. Suh, and U. C. De, A note on generalized Robertson-Walker space-times, Int. J. Geom. Meth. Mod. Phys. 13 (2016), 1650079 (9pp.).

[12] C. A. Mantica and L. G. Molinari, Riemann compatible tensors, Colloq. Math. 128 n.2 (2012) $197-210$.

[13] C. A. Mantica and L. G. Molinari, Weyl compatible tensors, Int. J. Geom. Meth. Mod. Phys. 11 (2014) 1450070 (15 pp).

[14] D. Lovelock, and H Rund, Tensors, Differential Forms and Variational Principles, Reprinted Edition (Dover, 1988).

[15] B-Y. Chen, K. Yano, Hypersurfaces of conformally flat spaces, Tensor (N.S.) 26 (1972), 318-322.

[16] M. Brozos-Vázquez, E. Garcia-Rio, and R. Vázquez-Lorenzo, Some remarks on locally conformally flat static space-times, J. Math. Phys. 46 ( 2005), 022501.

C. A. Mantica: I.I.S. Lagrange, Via L. Modignani 65, 20161, Milano, Italy - L. G. MoliNari (corresponding author): Physics Department, Università degli Studi di Milano and I.N.F.N. Sez. Milano, Via Celoria 16, 20133 Milano, Italy.

E-mail address: carloalberto.mantica@libero.it, luca.molinari@unimi.it 